\title{
On the Hyperbolicity of Einstein's and Other Gauge Field Equations ${ }^{\star}$
}

\author{
Helmut Friedrich \\ Universität der Bundeswehr Hamburg, Fachbereich Maschinenbau, Holstenhofweg 85, D-2000 \\ Hamburg 70, Federal Republic of Germany
}

\begin{abstract}
It is shown that Einstein's vacuum field equations (respectively the conformal vacuum field equations) in a frame formalism imply a symmetric hyperbolic system of "reduced" propagation equations for any choice of coordinate system and frame field (and conformal factor). Certain freely specifiable "gauge source" functions occurring in the reduced equations reflect the choice of gauge. Together with the initial data they determine the gauge uniquely. Their choice does not affect the isometry class (conformal class) of a solution of an initial value problem. By the same method symmetric hyperbolic propagation equations are obtained from other gauge field equations, irrespective of the gauge. Using the concept of source functions one finds that Einstein's field equation, considered as second order equations for the metric coefficients, are of wave equation type in any coordinate system.
\end{abstract}

\section{Introduction}

In this article rather general results on the hyperbolicity of Einstein's and other gauge field equations will be discussed. They were obtained by an analysis of the conformal vacuum field equations in the context of a specific initial value problem. The "conformal structure" of Einstein's vacuum field equations allows one to represent the conformal vacuum field equations

$$
\operatorname{Ric}\left(\Omega^{-2} g\right)=0
$$

for the "non-physical" metric $g$ and the "conformal factor" $\Omega$ by a system of first order partial differential equations which remains regular even where $\Omega$ vanishes [1]. By this property of the field equations it is possible to transform global "initial value" problems for Einstein's vacuum field equations, where the solutions are to be characterized by their limiting behaviour near past null infinity, into local initial

* Work supported by a Heisenberg-Fellowship of the Deutsche Forschungsgemeinschaft 
value problems for the conformal vacuum field equations with data being given on surfaces, which intersect or form part of null infinity. A crucial prerequisite for proving existence theorems for these local problems is the possibility of casting the regular conformal vacuum field equations, which are given in a frame formalism, into a form to which the theorems of the theory of partial differential equations apply. It turns out $[1,2,3]$, that in a certain geometric gauge of the coordinates and the frame field various characteristic and standard Cauchy problems for the vacuum and the regular conformal vacuum field equations can be formulated as initial value problems for quasilinear "symmetric hyperbolic" systems $[4,5]$. These are equations of the type

$$
A^{\mu} t_{, \mu}+b=0
$$

for a vector-valued unknown $t=t\left(x^{\mu}\right)$, with matrices $A^{\mu}$ and a vector-valued function $b$, which may depend on the coordinates $x^{\mu}$ and the unknown $t$, such that

$$
{ }^{T} \bar{A}^{\mu}=A^{\mu} ; \quad \exists \xi_{\mu} \text { with: } A^{\mu} \xi_{\mu} \text { is positive definite. }
$$

Extensive work has been done on symmetric hyperbolic systems, and existence theorems for the standard Cauchy problem have been worked out in detail and in great generality [6], which apply immediately to Cauchy problems for the conformal vacuum field equations [3].

Though the regular conformal vacuum field equations are well adapted to any situation where data are given at null infinity, the technique of reducing initial value problems for these equations to problems for symmetric hyperbolic systems as discussed in [1], does not apply to the particularly interesting "pure radiation problem" [7]. Here data are prescribed on a cone representing past null infinity. Its vertex, which represents past timelike infinity, is required to be smooth in the sense that the non-physical spacetime may be smoothly extended through it. Furthermore, the null curves on the cone are required to satisfy a certain completeness condition. For rather general data solutions of the conformal vacuum field equations near this "null cone at past timelike infinity" would yield solutions of Einstein's vacuum field equations which are "semiglobal" in the sense that they are past null and timelike geodesically complete. They would have an unambiguous interpretation as representing gravitational radiation, coming in from infinity interacting with itself and possibly going out to infinity again. It is clear that a thorough understanding of this problem, in particular of the behaviour of the solutions near spatial infinity and in the future, should enhance our insight into the effects and peculiarities of the nonlinear interactions of gravitational waves considerably.

The geometrical gauge used in [1] for the reduction of the field equations however, fails in this situation, since the geometrically defined coordinates and frame fields, suitably adapted to the cone, become singular at the vertex. This illustrates that it is desirable to increase the flexibility of the formalism by extending the reduction technique to more general classes of gauge conditions. The investigation of this question led to a result which not only applies to the pure radiation problem but which is of such generality that it is of interest independent of the original problem. It turns out that by a quite general procedure a symmetric 
hyperbolic system of propagation equations can be extracted from the conformal vacuum field equations, irrespective of the chosen coordinates, frame, and conformal factor.

Critical for obtaining the symmetric hyperbolic form is the observation, most easily made and stated in the spin frame formalism, that equations of the type

$$
\nabla_{b^{\prime}}^{b} f_{b \beta}=F_{b^{\prime} \beta}\left(f_{c \gamma}\right)
$$

imply symmetric hyperbolic systems. Here the unknown field $f_{b \beta}$ carries a spinor index $b$ and some multiindex $\beta$, which may be spinorial or of quite a different nature, and $F_{b^{\prime} \beta}$ is some smooth function. Important in (1.4) is the structure of the principal part

$$
e^{\mu b}{ }_{b^{\prime}} f_{b \beta, \mu}
$$

where $e_{a a^{\prime}}^{\mu}=\bar{e}_{a a^{\prime}}^{\mu}$ are the coefficients of a pseudo-orthonormal frame with spinor indices $a, a^{\prime}$ taking values 0,1 . Formally Eq. (1.4), written in the order

$$
\begin{aligned}
-\nabla^{b}{ }^{\prime} f_{b \beta} & =-F_{0^{\prime} \beta}\left(f_{c \gamma}\right), \\
\nabla^{b}{ }_{1}, f_{b \beta} & =F_{1^{\prime} \beta}\left(f_{c \gamma}\right) .
\end{aligned}
$$

are of the form (1.2), (1.3) with $\xi_{\mu}={\sigma_{\mu}}^{00^{\prime}}+\sigma_{\mu}{ }^{11^{\prime}}$, where $\sigma^{a a^{\prime}}$ is the frame of 1 -forms dual to $e_{a a^{\prime}}$. If, however, symmetries are required, e.g. $f_{b \beta}=f_{b c d . . . h}=$ $f_{(b c d . . . h)}$, the symmetric hyperbolic system is obtained in a slightly more complicated way (see (3.11)-(3.15) and the discussion in [8]).

The second basic ingredient in the reduction technique is the following idea of a "gauge source." In Sect. 2 it will be shown that on a given Lorentz-space the choice of a coordinate system and a frame field can always uniquely be characterized by suitable initial data and certain functions, called gauge sources, which in a sense represent the "pure gauge content" of the frame and the connection coefficients. For a given gauge the gauge sources can easily be calculated and, conversely, they can be arbitrarily prescribed to locally determine a new gauge. The interest in the gauge sources arises from the fact that by writing them explicitly into the field equations, one has to introduce compensating terms, which combine with the original terms in the equations to yield the form (1.5) of the principal part.

The class of solutions of the conformal vacuum field Eq. (1.1) is invariant under rescalings of the form

$$
(\Omega, g) \rightarrow(\hat{\Omega}, \hat{g})=\left(\Theta \Omega, \Theta^{2} g\right),
$$

where $\Theta$ is a positive function. In [1] this freedom has essentially been removed by the requirement that the Ricci scalar of the non-physical metric vanish. This leads already in quite simple cases to the difficulty that the conformal factor, which is obtained as part of the solution of the regular conformal field equations, will go to zero at places where it should not. It will be seen in Sect. 3 that it is possible to specify the Ricci scalar freely in an initial value problem for the conformal vacuum field equations, without destroying the symmetric hyperbolic form of the reduced propagation equations. Thus the Ricci scalar may be considered as the gauge source for the conformal factor.

Though the discussion in Sect. 2 of how to arrive at the symmetric hyperbolic 
form is quite general, there are problems with a straightforward application of this technique to Einstein's field equations with matter fields, if one wants to use a second order formalism. However, as will be shown in Sect. 3, the idea of the coordinate gauge source may be used to exhibit the hyperbolic nature of Einstein's equations when considered as second order equations for the metric coefficients, irrespective of the chosen coordinate system. The reduction of the equations by the harmonic gauge $[9,10]$ will then appear as a special case of a general procedure.

In view of the formal similarity of the vacuum field equations in the frame formalism with other gauge field equations, it is not surprising that the method can be used to cast the propagational part of the gauge field equations in any chosen gauge into the symmetric hyperbolic form. This is shown in Sect. 3, while in Sect. 2 the corresponding gauge source function is derived.

The discussion of the hyperbolic structure of Einstein's equations and of the conformal vacuum field equations given in this paper should prove useful in general arguments as well as in specific initial value problems. In the context of existence proofs the question of which gauge conditions should be chosen to prevent or postpone the development of a degeneracy or a blow-up of the coordinates and the frame now not only becomes particularly interesting but an answer should lead to consequences, since the evolution of the gauge can be controlled by the form of the gauge sources. They may be coupled to the geometric fields either algebraically or by imposing a system of partial differential equations, which is added to the reduced equations. A similar question is how to prescribe the Ricci scalar in an initial value problem for the conformal vacuum field equations such that the conformal factor will show the correct behaviour. It is an interesting but still open problem, which information on the global structure of the prospective solution spacetime has to be encoded into the sought for form of the Ricci scalar. Again, it may be helpful to allow the Ricci scalar itself to pick up the necessary information on the geometry by describing for it an appropriate coupling to the other fields.

\section{The Gauge Sources}

In the following the spin frame formalism, which reflects the desired structure of the equations most naturally, will be used freely in the notation of $[1,2]$. All functions will be smooth and the summation convention will hold.

On a four dimensional manifold with Lorentz-metric $g$ and metric connection $\nabla$, let $\left\{x^{\mu}\right\}_{\mu=0,1,2,3}$ be a local coordinate system and $\left(\omega_{a}\right)_{a=0,1}$ be a normalized (i.e. satisfying $\left(\omega_{a}, \omega_{b}\right)=\varepsilon_{a b}$ with $\varepsilon_{01}=1$, where $\varepsilon$ is the antisymmetric form on the spinor bundle) spinframe field, defined on the coordinate patch. The spinor fields $\omega_{a} \bar{\omega}_{a^{\prime}}$ correspond to a pseudoorthonormal frame field $\left(e_{a a^{\prime}}\right)_{a=0,1, a^{\prime}=0,1}$ with

$$
g\left(e_{a a^{\prime}}, e_{b b^{\prime}}\right)=\varepsilon_{a b} \varepsilon_{a^{\prime} b^{\prime}}, \quad \bar{e}_{a b^{\prime}}=e_{b a^{\prime}},
$$

which determines the frame $\sigma^{a a^{\prime}}$ of dual 1 -forms by

$$
\left\langle\sigma^{a a^{\prime}}, e_{b b^{\prime}}\right\rangle=\varepsilon_{b}{ }^{a} \varepsilon_{b^{\prime}}{ }^{a^{\prime}} .
$$

If everything is expressed with respect to $\omega_{a}$ respectively $e_{a a^{\prime}}$, the connection is 
represented by the connection coefficients $\Gamma_{a a^{\prime} b c}$ which satisfy

$$
\nabla_{a a^{\prime}} \omega_{b}:=\nabla_{e a a^{\prime}} \omega_{b}=\Gamma_{a a^{\prime} b}{ }^{c} \omega_{c} ; \quad \Gamma_{a a^{\prime} b c}=\Gamma_{a a^{\prime}(b c)} .
$$

The torsion tensor $t_{a a^{\prime}}{ }^{b b^{\prime}}{ }_{c c^{\prime}}$ is defined by

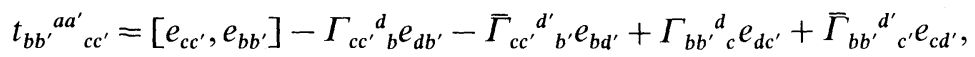

where the square brackets denote the commutator of vector fields. Applying the frame to the coordinate functions, one obtains the frame coefficients $e_{a a^{\prime}}\left(x^{\mu}\right)=$ : $e^{\mu}{ }_{a a^{\prime}}$, which have a dual interpretation. For fixed lower indices they are the components of a given vector field with respect to the coordinates $x^{\mu}$, for fixed upper index they represent the differential $d x^{\mu}=e_{a a^{\prime}}^{\mu} \sigma^{a a^{\prime}}$ with respect to the frame $e_{a a^{\prime}}$. With the second meaning one has

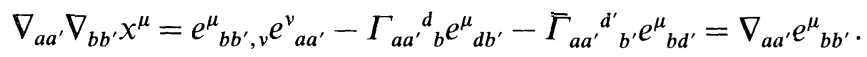

Applying (2.3) to the function $x^{\mu}$, using (2.4), and exploiting the antisymmetry of the torsion tensor one obtains an equivalent expression for (2.3) in the form of two equations

$$
t_{b a}^{f f^{\prime}} e_{f f^{\prime}}^{\mu}=\nabla_{(a}^{f^{\prime}} e_{b) f^{\prime}}^{\mu}, \quad t_{b^{\prime} a^{\prime}}^{f f^{\prime}} e_{f f^{\prime}}^{\mu}=\nabla_{\left(a^{\prime}\right.}^{f} e_{\left.b^{\prime}\right) f}^{\mu},
$$

where the brackets denote symmetrization, $t_{b c}^{f f^{\prime}}=\frac{1}{2} t_{b h^{\prime}}{ }^{f f^{\prime}{ }_{c}{ }^{\prime}}=t_{(b c)}^{f f^{\prime}}$, and $t_{b^{\prime} a^{\prime}}^{f f^{\prime}}$ is the complex conjugate of this, since $e_{a a^{\prime}}$ satisfies (2.1). Therefore (2.3) is in fact equivalent to each of Eq. (2.5) if $e_{a a^{\prime}}=\bar{e}_{a a^{\prime}}$. An important point here is, that the real-valued functions

$$
F^{\mu}=\frac{1}{2} \nabla^{f f^{\prime}} e_{f f^{\prime}}^{\mu}=\frac{1}{2} \nabla^{f f^{\prime}} \nabla_{f f^{\prime}} x^{\mu}
$$

drop out of Eq. (2.5), whence play no rôle in the definition of the torsion tensor.

The curvature spinor $r_{a b c c^{\prime} d d^{\prime}}$ is defined by

$$
\begin{aligned}
& r_{a b c c^{\prime} d d^{\prime}}+t_{c c^{\prime}} f^{\prime}{ }_{d d^{\prime}} \Gamma_{f f^{\prime} a b}=e_{d d^{\prime}}\left(\Gamma_{c c^{\prime} a b}\right)-\Gamma_{d d^{\prime}}{ }_{c} \Gamma_{f c^{\prime} a b}-\bar{\Gamma}_{d d^{\prime}}{ }^{f^{\prime}}{ }_{c^{\prime}} \Gamma_{c f^{\prime} a b}-\Gamma_{d d^{\prime}}{ }_{b} \Gamma_{c c^{\prime} a f} \\
& -\left\{e_{c c^{\prime}}\left(\Gamma_{d d^{\prime} a b}\right)-\Gamma_{c c^{\prime}}{ }^{f}{ }_{d} \Gamma_{f d^{\prime} a b}-\bar{\Gamma}_{c c^{\prime}}{ }^{f^{\prime}{ }_{d^{\prime}}} \Gamma_{d f^{\prime} a b}-\bar{\Gamma}_{c c^{\prime}}{ }^{f^{\prime}{ }_{b^{\prime}}} \Gamma_{d d^{\prime} a f}\right\} .
\end{aligned}
$$

To simplify the following expressions it is convenient to define

$$
\begin{aligned}
\nabla_{a a^{\prime}} \Gamma_{b b^{\prime} c d}:= & e_{a a^{\prime}}\left(\Gamma_{b b^{\prime} c d}\right)-\Gamma_{a a^{\prime}}{ }_{b} \Gamma_{f b^{\prime} c d}-\bar{\Gamma}_{a a^{\prime}}{ }^{f^{\prime}}{ }_{b^{\prime}} \Gamma_{b f^{\prime} c d}-\Gamma_{a a^{\prime}}{ }_{c}{ }_{c} \Gamma_{b b^{\prime} f d} \\
& -\Gamma_{a a^{\prime}}{ }^{f}{ }_{d} \Gamma_{b b^{\prime} c f},
\end{aligned}
$$

which is the same expression which one obtains for the covariant derivative of a spinor field with the same index structure as that of the $\Gamma$ 's, which takes with respect to the given spinframe the same values as the $\Gamma$ 's. Using this definition and taking into account the antisymmetry of the curvature spinor in the last index pairs, Eq. (2.7) finds equivalent expression in the two equations

$$
\begin{aligned}
& r_{d c}^{a b}+t_{d c}^{f f^{\prime}} \Gamma_{f f^{\prime}}^{a b}=\nabla_{(c} e^{e^{\prime}} \Gamma_{d) e^{\prime}}{ }^{a b}+\Gamma_{(c}{ }^{e^{\prime} f(a} \Gamma_{d) e^{\prime}}{ }^{b)}{ }_{f}, \\
& r^{a b}{ }_{d^{\prime} c^{\prime}}+t_{d^{\prime} c^{\prime}}^{f f^{\prime}} \Gamma_{f f^{\prime}}^{a b}=\nabla_{\left(c^{\prime}\right.}^{e} \Gamma_{\left.d^{\prime}\right) e}{ }^{a b}+\Gamma_{\left(c^{\prime}\right.}^{e}{ }^{f(a} \Gamma_{\left.d^{\prime}\right) e}{ }^{b)}{ }_{f} \text {, }
\end{aligned}
$$

where $r_{a b d c}=\frac{1}{2} r_{a b d f^{\prime} c^{\prime}}{ }^{\prime}, r_{a b d^{\prime} c^{\prime}}=\frac{1}{2} r_{a b f d^{\prime}}{ }^{\prime} c^{\prime}$. Here one recognizes that the quantity

$$
F_{a b}:=\nabla^{d d^{\prime}} \Gamma_{d d^{\prime} a b},
$$


which satisfies

$$
F_{a b}=F_{(a b)}=e^{d d^{\prime}}\left(\Gamma_{d d^{\prime} a b}\right)-\left(\Gamma_{f}^{f d^{\prime} d}+\bar{\Gamma}_{f^{\prime}}^{d f^{\prime} d^{\prime}}\right) \Gamma_{d d^{\prime} a b}
$$

drops out of Eqs. (2.9), (2.10), respectively (2.7), whence has no effect on the definition of the curvature tensor. While it is obvious that $F^{\mu}$ is obtained by applying a gauge invariant operator to the gauge dependent quantity $x^{\mu}$, it is not so obvious that $F_{a b}$ is defined by a similar procedure. For fixed $a, b$ the quantities $\varepsilon\left(\nabla_{d d^{\prime}}, \omega_{a}, \omega_{b}\right)$ represent the components of a 1 -form. Taking its divergence and observing (2.2), (2.12), one obtains

$$
\nabla^{d d^{\prime}}\left(\varepsilon\left(\nabla_{d d^{\prime}} \omega_{a}, \omega_{b}\right)\right)=F_{a b}
$$

Equivalently one has

$$
\nabla^{\mu}\left(g\left(\nabla_{\mu} e_{a a^{\prime}}, e_{b b^{\prime}}\right)\right)=\varepsilon_{a^{\prime} b^{\prime}} F_{a b}+\varepsilon_{a b} \bar{F}_{a^{\prime} b^{\prime}} .
$$

Another interpretation of $F_{a b}$ which is immediately read off (2.12) is

$$
\delta \vartheta^{a}{ }_{b}=-F^{a}{ }_{b},
$$

where $\vartheta^{a}{ }_{b}=\Gamma_{c c^{\prime} b}{ }^{a} \sigma^{c c^{\prime}}$ is the pull-back of the connection form on the bundle of spin frames by the local section defined by $\omega_{a}$ and $\delta$ is the codifferential. The functions $F^{\mu}$, respectively $F_{a b}$, will be called "coordinate gauge source," respectively "frame gauge source." The reason for this name will become clear later, when it will be shown that these functions act as source terms in the reduced vacuum field equations (Eqs. (3.11)-(3.13) with $\Omega \equiv 1, \phi_{a b a^{\prime} b^{\prime}} \equiv 0, \Lambda \equiv 0$ ) and determine the coordinate system and the frame field, without affecting the geometry.

Suppose real valued functions $\hat{F}^{\mu}$ and complex valued functions $\hat{F}_{a b}=\widehat{F}_{(a b)}$, defined on $\mathbb{R}^{4}$, are given arbitrarily. Then on the given space-time there exists a coordinate system $\hat{x}^{\mu}$ and a spin frame $\hat{\omega}_{a}$, such that in the coordinate system $\hat{x}^{\mu}$ the corresponding gauge sources are just given by $\hat{F}^{\mu}, \hat{F}_{a b}$. Reading Eqs. (2.6), (2.13) as differential equations suggests obtaining $\hat{x}^{\mu}, \hat{\omega}_{a}$ as solution of the system of semilinear wave equations

$$
\begin{gathered}
\nabla^{f f^{\prime}} \nabla_{f f^{\prime}} \hat{x}^{\mu}=2 \hat{F}^{\mu}\left(\hat{x}^{v}\right) \quad \mu=0,1,2,3, \\
\nabla^{f f^{\prime}}\left(\varepsilon\left(\nabla_{f f^{\prime}} \hat{\omega}_{a}, \hat{\omega}_{b}\right)\right)=\hat{F}_{a b}\left(\hat{x}^{v}\right) \quad a, b=0,1 .
\end{gathered}
$$

For suitably given initial data these equations determine $\hat{x}^{\mu}, \hat{\omega}_{a}$ uniquely near the initial surface. Equation (2.16) can be solved separately. Notice that on the right of (2.16) a function of the unknowns appears, not a source term, whereas, once $\hat{x}^{\mu}$ is known, the right member of (2.17) acts a source term. The structure of (2.17) becomes clearer by writing it in a more explicit way. Let $t^{b}{ }_{a}$ be the components of $\hat{\omega}_{a}$ with respect to the basis $\omega_{b}$, then (2.17) takes the form

$$
\nabla_{\mu}\left(\left(\nabla^{\mu} t_{a}^{c}\right) \varepsilon_{c d} t_{b}^{d}\right)=\hat{F}_{a b}
$$

respectively

$$
\nabla_{\mu} \nabla^{\mu} t^{c}{ }_{a}=-\varepsilon_{e f} \nabla^{\mu} t^{e}{ }_{a} \nabla_{\mu} t_{b}^{f} t^{-1_{b}}{ }_{h} \varepsilon^{c h}+\hat{F}_{a b} t^{-1_{b}}{ }_{h} \varepsilon^{c h} .
$$

From (2.18) and the symmetry of $\hat{F}_{a b}$ follows

$$
\nabla_{\mu} \nabla^{\mu}\left(\varepsilon_{a b} t^{a}{ }_{c} t^{b}{ }_{d}\right)=0,
$$


from which one concludes: if the initial data are given such that

$$
\varepsilon_{a b} t^{a}{ }_{c} t_{d}^{b}=\varepsilon_{c d}, \quad \nabla_{\mu}\left(\varepsilon_{a b} t^{a}{ }_{c} t_{d}^{b}\right)=0
$$

hold on the initial surface, then the solution of (2.18) satisfies (2.19) everywhere near this surface, whence $\operatorname{det}\left(t^{a}{ }_{b}\right)=1$ and $\hat{\omega}_{a}$ is in fact a normalized spin basis.

Using (2.6), respectively (2.11), Eqs. (2.5), respectively (2.9), (2.10) can be written:

$$
\begin{gathered}
\nabla_{a} f^{\prime} e_{b f^{\prime}}^{\mu}+\varepsilon_{a b} F^{\mu}=t_{b a}^{h h^{\prime}} e_{h h^{\prime}}^{\mu}, \\
\nabla_{a^{\prime}}^{f} e_{f b^{\prime}}^{\mu}+\varepsilon_{a^{\prime} b^{\prime}} F^{\mu}=t_{b^{\prime} a^{\prime}}^{h h^{\prime}} e_{h h^{\prime}}^{\mu}, \\
2 \nabla_{c}{ }^{f^{\prime}} \Gamma_{d f^{\prime}}{ }^{a b}+\Gamma_{(c}{ }^{e^{\prime} f(a} \Gamma_{d) e^{\prime}{ }_{f}{ }_{f}+\varepsilon_{c d}}+\varepsilon^{a b}=2\left(r^{a b}{ }_{d c}+t_{d c}^{h h^{\prime}} \Gamma_{h h^{\prime}}{ }^{a b}\right), \\
2 \nabla^{f}{ }_{c^{\prime}} \Gamma_{f d^{\prime}}{ }^{a b}+\Gamma^{e}{ }_{\left({ }^{\prime}{ }^{\prime}\right.}{ }^{f(a} \Gamma_{\left.d^{\prime}\right) e}{ }^{b)}{ }_{f}+\varepsilon_{c^{\prime} d^{\prime}} F^{a b}=2\left(r^{a b}{ }_{d^{\prime} c^{\prime}}+t_{d^{\prime} c^{\prime}}^{h h^{\prime}} \Gamma_{h h^{\prime}}{ }^{a b}\right) .
\end{gathered}
$$

Hence by introducing explicitly the source functions, the desired form of the differential operator on the left is obtained. In a general space-time one has

$$
r_{a b d c}=-\psi_{a b c d}-\Lambda\left(\varepsilon_{a c} \varepsilon_{b d}+\varepsilon_{a b} \varepsilon_{b c}\right), \quad r_{a b d^{\prime} c^{\prime}}=-\phi_{a b c^{\prime} d^{\prime}}
$$

with

$$
\psi_{a b c d}=\psi_{(a b c d)}, \quad 24 \Lambda=R=\bar{R}, \quad 2 \phi_{a b a^{\prime} b^{\prime}}=2 \phi_{(a b)\left(a^{\prime} b^{\prime}\right)}=2 \Phi_{a b a^{\prime} b^{\prime}}
$$

being the Weyl spinor, respectively the Ricci scalar, respectively the traceless part of the Ricci tensor. The field equations will be formulated as conditions on these fields and on $t_{b a}^{h h^{\prime}}$ respectively $t_{b^{\prime} a^{\prime}}^{h h^{\prime}}$. For given right-hand sides, the pair of Eqs. (2.21), (2.23) forms a symmetric hyperbolic system if $e_{a a^{\prime}}=\bar{e}_{a a^{\prime}}$ holds in $\nabla_{a a^{\prime}}$. For suitable initial data and given gauge sources it determines the frame and the connection coefficients uniquely. Then the left-hand sides of all equations are determined. A similar remark holds for the pair (2.20), (2.22). In order that this does not lead to contradictions, the conditions on the curvature and the torsion tensors have to be given in accordance with the Bianchi identities, which are the compatibility conditions for the equations above.

On a Lorentz manifold with metric $g_{\mu \nu}$ and Levi-Civita connection $\nabla$ the pure gauge field equations for the gauge potential $A_{\mu}$ and the gauge field $F_{\mu \nu}$ read

$$
\nabla_{\mu} A_{v}-\nabla_{v} A_{\mu}-\left[A_{\mu}, A_{v}\right]+F_{\mu \nu}=0, \quad \nabla_{\mu} F_{\nu}^{\mu}-\left[A_{\mu}, F^{\mu}{ }_{\nu}\right]=0,
$$

where the bracket denotes the Lie product. The fields $A_{\mu}, F_{\mu \nu}$ take their values in the Lie algebra of some gauge Group $G$, which for the sake of definiteness will be assumed to be some $\mathrm{SU}(n), n \geqq 2$. In the following it may be understood that all fields are given with respect to the standard representation of this group, however matrix indices will be suppressed. Using the spin frame formalism as before, representing the gauge field by $\varepsilon_{a b} \bar{\varphi}_{a^{\prime} b^{\prime}}+\varepsilon_{a^{\prime} b^{\prime}} \varphi_{a b}$, where $\varphi_{a b}=\varphi_{(a b)}$ takes its values in $\operatorname{su}(n)$, and defining

$$
\begin{aligned}
l_{a a^{\prime} b b^{\prime}} & =\nabla_{a a^{\prime}} A_{b b^{\prime}}-\nabla_{b b^{\prime}} A_{a a^{\prime}}-\left[A_{a a^{\prime}}, A_{b b^{\prime}}\right]+\left(\varphi_{a b} \varepsilon_{a^{\prime} b^{\prime}}+\bar{\varphi}_{a^{\prime} b^{\prime}} \varepsilon_{a b}\right), \\
h_{c^{\prime} c} & =\nabla^{f}{ }_{c^{\prime}} \varphi_{c f}-\left[A_{c^{\prime}}^{f}, \varphi_{c f}\right],
\end{aligned}
$$

Eq. (2.25) can be represented equivalently by

$$
l_{a a^{\prime} b b^{\prime}}=0, \quad h_{c^{\prime} c}=0,
$$


where the Bianchi identities have been used. The first of these equations is equivalent to

$$
l_{a b}=l_{a b f^{\prime}}^{f^{\prime}}=0, \quad l_{a^{\prime} b^{\prime}}=l_{a^{\prime} f b^{\prime}}^{f}=0 .
$$

These two equations are equivalent since $A_{a a^{\prime}}=\bar{A}_{a a^{\prime}}$, where ${ }^{-}$denotes the spinor calculus complex conjugation. There are two ways of writing $l_{a b}=l_{(a b)}$,

$$
l_{b a}=2 \nabla_{(a}{ }^{f^{\prime}} A_{b) f^{\prime}}-\left[A_{a}{ }^{f^{\prime}}, A_{b f^{\prime}}\right]-2 \varphi_{a b}=2 \nabla_{a}{ }^{f^{\prime}} A_{b f^{\prime}}+\varepsilon_{a b} B-\left[A_{a}{ }^{f^{\prime}}, A_{b f^{\prime}}\right]-2 \varphi_{a b} .
$$

The first form shows that $l_{a b}$, whence by (2.28) the definition of $\varphi_{a b}$, is independent of the "gauge source"

$$
B=\nabla^{f f^{\prime}} A_{f f^{\prime}} .
$$

The second form shows that by introducing the source function again the desired form of the differential operator is obtained. Similar remarks hold for $l_{a^{\prime} b^{\prime}}$, where the same source function turns up. Equations (2.15) and (2.30) are seen to be perfect analogues of each other. As before, one finds that locally the gauge source can be given arbitrarily to determine together with suitable initial data a new gauge. On the given space time let $\widehat{B}=\widehat{\hat{B}}$ be some su(n)-valued function. Assuming that $\hat{B}=\nabla^{f f^{\prime}} \hat{A}_{f f^{\prime}}$ where $\hat{A}_{f f^{\prime}}$ is a gauge potential related to $A_{f f^{\prime}}$ by a gauge transformation $s=s(x)$,

$$
\hat{A}_{f f^{\prime}}=s^{-1}\left(\nabla_{f f^{\prime}} s+A_{f f^{\prime}} s\right)
$$

one finds that $s$ must satisfy the semilinear wave equation

$$
\nabla^{f f^{\prime}} \nabla_{f f^{\prime}} s=s \hat{B}-B s+\left(\nabla^{f f^{\prime}} s\right) s^{-1}\left(\nabla_{f f^{\prime}} s\right)+\left(\nabla^{a a^{\prime}} s\right) s^{-1} A_{a a^{\prime}} s-A_{a a^{\prime}} \nabla^{a a^{\prime}} s
$$

For initial data $s, \nabla_{f f^{\prime}} s$, which are given on a suitable initial surface such that $s \in \mathrm{SU}(n), s^{-1} \nabla_{f f^{\prime}} s \in \mathrm{Su}(n)$, Eq. (2.32) determines a unique gauge transformation near the initial surface. That $s$ takes its values in $\mathrm{SU}(n)$ follows from $(2.32)$, since this equation implies equations of the type

$$
\begin{aligned}
\nabla^{f f^{\prime}} \nabla_{f f^{\prime}}\left({ }_{\mathrm{S}}^{*} \cdot \mathrm{s}\right) & =(\stackrel{*}{\mathrm{~S}} \cdot \mathrm{s}) \hat{B}-B(\stackrel{*}{\mathrm{~S}} \cdot \mathrm{s})+H_{a a^{\prime}} \nabla^{a a^{\prime}}(\stackrel{*}{\mathrm{~S}} \cdot \mathrm{s}), \\
\nabla^{f f^{\prime}} \nabla_{f f^{\prime}}(\operatorname{det} s) & =K_{a a^{\prime}} \nabla^{a a^{\prime}}(\operatorname{det}(s)),
\end{aligned}
$$

where ${ }^{*}$ denotes the hermitian conjugate.

\section{The Hyperbolic Propagation Equations}

The "regular conformal vacuum field equations" for the unknown

read

$$
t=\left(e_{a a^{\prime}}^{\mu}, \Gamma_{a a^{\prime} b c^{\prime}}, \varphi_{a b c d}, \phi_{a b a^{\prime} b^{\prime}}, \Omega, \Sigma_{a a^{\prime}} s\right)
$$

$$
\begin{aligned}
t_{a a^{\prime}}{ }^{b b^{\prime}{ }_{c c^{\prime}}} & =0 \\
r_{a b c c^{\prime} d d^{\prime}} & =-\Omega \varphi_{a b c d} \varepsilon_{c^{\prime} d^{\prime}}-\phi_{a b c^{\prime} d^{\prime}} \varepsilon_{c d}+\Lambda \varepsilon_{c^{\prime} d^{\prime}}\left(\varepsilon_{a c} \varepsilon_{b d}+\varepsilon_{a d} \varepsilon_{b c}\right), \\
\nabla_{b^{\prime}}^{f} \varphi_{a b c f} & =0 \\
\nabla_{a}^{f^{\prime}} \phi_{b c d^{\prime} f^{\prime}} & =\varphi_{a b c f} \Sigma^{f}{ }_{d^{\prime}}-2 \varepsilon_{a(c} \nabla_{b) d^{\prime}} \Lambda, \\
\nabla_{a a^{\prime}} \Omega & =\Sigma_{a a^{\prime}},
\end{aligned}
$$




$$
\begin{aligned}
\nabla_{a a^{\prime}} \Sigma_{b b^{\prime}} & =-\Omega \phi_{a b a^{\prime} b^{\prime}}+s \varepsilon_{a b^{\prime}} \varepsilon_{a^{\prime} b^{\prime}}, \\
\nabla_{a a^{\prime}} S & =-\phi_{a b a^{\prime} b^{\prime}} \Sigma^{b b^{\prime}}-2 \Lambda \Sigma_{a a^{\prime}}-\Omega \nabla_{a a^{\prime}} \Lambda, \\
0 & =2 \Omega s-\Sigma_{a a^{\prime}} \Sigma^{a a^{\prime}}+2 \Omega^{2} \Lambda .
\end{aligned}
$$

These equations are "conformally invariant" in the sense that under the rescaling (1.7) and the implied transformations of the unknowns, the equations retain their form. They are more general than those given in [1], since the choice of the real valued function $\Lambda$ is left open. This function, which may be specified freely, will be considered as gauge source for the conformal factor. The feasibility of this interpretation is suggested by the transformation law

$$
\nabla_{k} \nabla^{k} \Theta=4\left(\Theta \Lambda(g)-\Theta^{3} \Lambda\left(\Theta^{2} g\right)\right)
$$

of $\Lambda=\Lambda(g)$ under the rescaling (1.7). It shows that $\Lambda\left(\Theta^{2} g\right)$ can be given freely and $\Theta$ will be obtained as a solution of a semilinear wave equation for suitable initial data.

Equations (3.1)-(3.8) have been discussed in [1,2] and only a few remarks will be made here. The spinor field $\varphi_{a b c d}=\Omega^{-1} \psi_{a b c d}$ is the rescaled Weyl spinor, therefore Eqs. (3.1), (3.2) are those discussed in Sect. 2. Equation (3.3) is the vacuum Bianchi identity for the physical fields rewritten in terms of the non-physical fields. Equation (3.4) is obtained from the Bianchi identity and Eq. (3.3). By (3.5) $\Sigma_{a a^{\prime}}$ is defined and by contraction of (3.6) one finds that $s=\frac{1}{4} \nabla_{a a^{\prime}} \nabla^{a a^{\prime}} \Omega$. Equation (3.6) is the traceless part of (1.1), the trace being given by (3.8). Equation (3.7) is derived from (3.1)-(3.6) because it has a "nice" principal part and it can be shown that (3.8) is implied by the other equations, if (3.8) holds at one point. To obtain a solution of (1.1) it is thus sufficient to solve (3.1)-(3.7) for suitable data, which in particular satisfy (3.8). Equations (3.1)-(3.3) with $\Omega \equiv 1 ; \phi_{a b a^{\prime} b^{\prime}} \equiv 0, \Lambda \equiv 0$ are just Einstein's vacuum field equations.

The symmetric hyperbolic system deduced from (3.1)-(3.7) will look more complicated than it might be expected from the preceding discussion. Since $e^{\mu}{ }_{a a^{\prime}}$ is obtained as solution of the field equations, one must make sure that it is possible to show that in fact one has $e_{a a^{\prime}}^{\mu}=\bar{e}_{a a^{\prime}}^{\mu}$. This requirement motivates the form of Eq. (3.11), which in turn suggests the form of (3.12). The principal parts of (3.3), (3.4) are of the form (1.5). However, the required symmetries of the fields $\varphi_{a b c d}, \phi_{a b a^{\prime} b^{\prime}}$ imply an overdeterminedness of Eqs. (3.3), (3.4), which is removed by the form of Eqs. (3.13), (3.14). It may be remarked that uniqueness results may be derived irrespective of such considerations.

Defining

$$
\gamma_{c d}^{a b}:=\frac{1}{2} \Gamma_{(c}^{e^{\prime} f(a} \Gamma_{d) e^{\prime}}^{b)}, \quad \gamma_{c^{\prime} d^{\prime}}^{a b}:=\frac{1}{2} \Gamma_{\left(e^{\prime}\right.}^{e}{ }^{f(a} \Gamma_{\left.d^{\prime}\right) e}{ }_{f}^{b)},
$$

and taking into account Eqs. (2.20)-(2.23), the following system of "reduced propagation equations" can be derived from (3.1)-(3.7):

$$
\begin{aligned}
& 2 \nabla_{00^{\prime}} e_{11^{\prime}}^{\mu}-\nabla_{01^{\prime}} e_{10^{\prime}}^{\mu}-\nabla_{10^{\prime}} e_{01^{\prime}}^{\mu}-2 F^{\mu}=0, \\
& \left(\nabla_{00^{\prime}}+\nabla_{11^{\prime}}\right) e_{10^{\prime}}^{\mu}-\nabla_{10^{\prime}} e_{11^{\prime}}^{\mu}-\nabla_{10^{\prime}} e_{00^{\prime}}^{\mu}=0, \\
& \left(\nabla_{00^{\prime}}+\nabla_{11^{\prime}}\right) e_{01^{\prime}}^{\mu}-\nabla_{01^{\prime}} e_{00^{\prime}}^{\mu}-\nabla_{01^{\prime}} e_{11^{\prime}}^{\mu}=0, \\
& 2 \nabla_{11^{\prime}} e_{00^{\prime}}^{\mu}-\nabla_{10^{\prime}} e_{01^{\prime}}^{\mu}-\nabla_{01^{\prime}} e_{10^{\prime}}^{\mu}-2 F^{\mu}=0,
\end{aligned}
$$




$$
\begin{aligned}
& 2 \nabla_{00^{\prime}} \Gamma_{11^{\prime}}{ }^{a b}-\nabla_{01^{\prime}} \Gamma_{10^{\prime}}{ }^{a b}-\nabla_{10^{\prime}} \Gamma_{01^{\prime}}{ }^{a b}-\gamma_{10}^{a b}-\gamma_{1^{\prime} 0^{\prime}}^{a b}-F^{a b} \\
& =\Omega \varphi^{a b}{ }_{01}-2 \Lambda \varepsilon_{0}{ }^{(a} \varepsilon_{1}{ }^{b)}+\phi^{a b}{ }_{0^{\prime} 1^{\prime}}, \\
& \left(\nabla_{00^{\prime}}+\nabla_{11^{\prime}}\right) \Gamma_{10^{\prime}}{ }^{a b}-\nabla_{10^{\prime}} \Gamma_{11^{\prime}}{ }^{a b}-\nabla_{10^{\prime}} \Gamma_{00^{\prime}}{ }^{a b}+\gamma_{11}^{a b}-\gamma_{0^{\prime} 0^{\prime}}^{a b} \\
& =-\Omega \varphi^{a b}{ }_{11}+2 \Lambda \varepsilon_{1}{ }^{a} \varepsilon_{1}{ }^{b}+\phi^{a b}{ }_{0^{\prime} 0^{\prime},} \\
& \left(\nabla_{00^{\prime}}+\nabla_{11^{\prime}}\right) \Gamma_{01^{\prime}}{ }^{a b}-\nabla_{01^{\prime}} \Gamma_{00^{\prime}}{ }^{a b}-\nabla_{01^{\prime}} \Gamma_{11^{\prime}}{ }^{a b}-\gamma_{00}^{a b}+\gamma_{1^{\prime} 1^{\prime}}{ }^{a b} \\
& =\Omega \varphi^{a b}{ }_{00}-2 \Lambda \varepsilon_{0}{ }^{a} \varepsilon_{0}{ }^{b}-\phi^{a b}{ }_{1{ }^{\prime} 1^{\prime}}, \\
& 2 \nabla_{11^{\prime}} \Gamma_{00^{\prime}}{ }^{a b}-\nabla_{10^{\prime}} \Gamma_{01^{\prime}}{ }^{a b}-\nabla_{01}, \Gamma_{10^{\prime}}{ }^{a b}+\gamma_{01}^{a b}+\gamma_{00^{\prime}{ }^{\prime}{ }^{a b}-F^{a b}} \\
& =-\Omega \varphi^{a b}{ }_{10}+2 \Lambda \varepsilon_{1}{ }^{(a} \varepsilon_{0}{ }^{b)}-\phi^{a b}{ }_{1^{\prime} 0^{\prime}}, \\
& -\nabla^{f}{ }_{0^{\prime}} \varphi_{111 f}=0 \text {, } \\
& -\nabla_{0^{\prime}}^{f} \varphi_{a b 0 f}+\nabla^{f}{ }_{1}^{\prime} \varphi_{a b 1 f}=0 \text { in the order } a b=11,10,00 \text {, } \\
& \nabla^{f}{ }_{1}, \varphi_{000 f}=0, \\
& -\nabla_{0}^{f^{\prime}} \phi_{b c 1^{\prime} f^{\prime}}=-\varphi_{0 b c f} \Sigma^{f}{ }_{1^{\prime}}+2 \varepsilon_{0(b} \nabla_{c) 1^{\prime}} \Lambda, \\
& -\nabla_{0} f^{\prime} \phi_{b c 0^{\prime} f^{\prime}}+\nabla_{1} f^{f^{\prime}} \phi_{b c 1^{\prime} f^{\prime}}=-\varphi_{0 b c f} \Sigma^{f_{0^{\prime}}}+\varphi_{1 b c f} \Sigma^{f}{ }_{1^{\prime}}+2\left(\varepsilon_{0(b} \nabla_{c) 0^{\prime}} \Lambda\right. \\
& \left.-\varepsilon_{1(b} \nabla_{c) 1}, \Lambda\right) \text {, } \\
& \nabla_{1}^{f^{\prime}} \phi_{b c 0^{\prime} f}=\varphi_{1 b c f} \Sigma^{f}{ }_{0^{\prime}}-2 \varepsilon_{1(b} \nabla_{c) 0^{\prime}} \Lambda \text {, } \\
& \left(\nabla_{00^{\prime}}+\nabla_{11^{\prime}}\right) \Omega=\Sigma_{00^{\prime}}+\Sigma_{11^{\prime}}, \\
& \left(\nabla_{00^{\prime}}+\nabla_{11^{\prime}}\right) \Sigma_{b b^{\prime}}=-\Omega\left(\phi_{0 b 0^{\prime} b^{\prime}}+\phi_{1 b 1^{\prime} b^{\prime}}\right)+s\left(\varepsilon_{0 b^{\prime}} \varepsilon_{0^{\prime} b^{\prime}}+\varepsilon_{1 b^{\prime}} \varepsilon_{1^{\prime} b^{\prime}}\right), \\
& \left(\nabla_{00^{\prime}}+\nabla_{11^{\prime}}\right) s=-\left(\phi_{0 b 0^{\prime} b^{\prime}}+\phi_{1 b 1^{\prime} b^{\prime}}\right) \Sigma^{b b^{\prime}}-2 \Lambda\left(\Sigma_{00^{\prime}}+\Sigma_{11^{\prime}}\right) \\
& -\Omega\left(\nabla_{00}, \Lambda+\nabla_{11}, \Lambda\right) \text {. }
\end{aligned}
$$

In these equations it is understood that $F^{\mu}=\bar{F}^{\mu}, F_{a b}=F_{(a b)}, \Lambda=\bar{\Lambda}$, are given smooth functions of four variables and that in $\nabla_{a a^{\prime}}$ the frame coefficients $e^{\mu}{ }_{a a^{\prime}}$ are replaced by $\frac{1}{2}\left(e_{a a^{\prime}}^{\mu}+\bar{e}_{a a^{\prime}}^{\mu}\right)$. Furthermore $e_{a a^{\prime}}^{\mu}, \phi_{a b a^{\prime} b^{\prime}}$ are considered as complex-valued functions where the reality conditions $e_{a a^{\prime}}^{\mu}=\bar{e}_{a a^{\prime}}$ and $\phi_{a b a^{\prime} b^{\prime}}=\bar{\phi}_{a b a^{\prime} b^{\prime}}$ are "forgotton" (hence have to be shown for a solution of (3.11)-(3.15)). Then (3.11)-(3.15) represent a symmetric hyperbolic system of partial differential equations for the unknown $t$. It may be pointed out that the type of nonlinearity of the field equations has not been changed by the introduction of the gauge source fields, and that the latter now act as source terms in the reduced equations.

The system (3.11)-(3.15) may be used to obtain existence results for various characteristic and standard Cauchy problems, each of which requires a separate discussion of Eqs. (3.1)-(3.8) on the initial surface. The following proposition will be formulated to cover all these cases without going into the analysis of the constraint equations, which is of no interest here.

Proposition. For functions $F^{\mu}, F_{a b}, \Lambda$ given on $\mathbb{R}^{4}$ and data given on some initial surface, let $t=\left(e_{a a^{\prime}}^{\mu}, \Gamma_{a a^{\prime} b c^{\prime}}, \varphi_{a b c d^{\prime}}, \phi_{a b a^{\prime} b^{\prime}}, \Omega, \Sigma_{a a^{\prime}}, s\right)$ be a solution of the reduced 
Eqs. (3.11)-(3.15). Ift satisfies the conformal vacuum field Eqs. (3.1)-(3.8) on the initial surface, then $t$ satisfies these equations in fact in the whole domain of dependence of the initial surface in the space-time defined by $t$.

Remark. It is important to know what will happen to the solutions of (3.11)-(3.15) if the source functions are changed. This will be described here for the case of a standard Cauchy problem for Eqs. (3.11)-(3.15), where data are prescribed on a spacelike hypersurface $S$. The constraint equations implied on $S$ by the conformal vacuum field equations and the way data have to be given such that a solution of (3.11)-(3.15) for these data satisfy (3.1)-(3.8) on $S$, have been discussed in [3]. Here it will be assumed that $t$ is a solution for suitable data on $S$ of Eqs. (3.1)-(3.8), hence in particular of (3.11)-(3.15), with some gauge source functions $F^{\mu}, F_{a b}, \Lambda$. Three types of transformation will be considered in the following:

(i) coordinate transformations $x^{\mu} \rightarrow \hat{x}^{\mu}\left(x^{\nu}\right)$, under which one has

$$
e_{a a^{\prime}}^{\mu} \rightarrow \frac{\partial \hat{x}^{\mu}}{\partial x^{v}} e^{v} a^{\prime},
$$

and the functions given by $t$ are expressed as functions of $\hat{x}^{\mu}$;

(ii) rotations of the frame $\omega_{a} \rightarrow \omega_{b} t^{b}{ }_{a}$ with $\operatorname{det}\left(t^{a}{ }_{b}\right)=1$, under which one has the transitions

$$
\begin{aligned}
& e^{\mu}{ }_{a a^{\prime}} \rightarrow e^{\mu}{ }_{b b} t^{b}{ }_{a} t^{b^{\prime}}{ }_{a^{\prime}},
\end{aligned}
$$

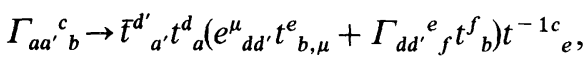

$$
\begin{aligned}
& \varphi_{a b c d} \rightarrow \varphi_{\text {efgh }} t^{e} t^{f}{ }_{b} t^{g}{ }_{c} t^{h},
\end{aligned}
$$

and similar transformations of the other fields given by $t$;

(iii) rescalings of the type (1.7), which, if the spinframe is rescaled in the form $\omega_{a} \rightarrow \Theta^{-1 / 2} \omega_{a}$, imply the transformations

$$
\begin{aligned}
& e^{\mu}{ }_{a a^{\prime}} \rightarrow \Theta^{-1} e^{\mu}{ }_{a a^{\prime}}, \\
& \Gamma_{a a^{\prime} b c} \rightarrow \Theta^{-1} \Gamma_{a a^{\prime} b c}+\Theta^{-2} \varepsilon_{a(b} \nabla_{c) a^{\prime}} \Theta, \\
& \varphi_{a b c d} \rightarrow \Theta^{-3} \varphi_{a b c d}, \\
& \phi_{a b c^{\prime} b^{\prime}} \rightarrow \Theta^{-2} \phi_{a b a^{\prime} b^{\prime}}-\Theta^{-3}\left(\nabla_{a a^{\prime}} \nabla_{b b^{\prime}} \Theta-\frac{1}{4} \varepsilon_{a b} \varepsilon_{a^{\prime} b^{\prime}}, \nabla_{f f^{\prime}} \nabla^{f f^{\prime}} \Theta\right) \\
& +2 \Theta^{-4}\left(\nabla_{a a^{\prime}} \Theta \nabla_{b b^{\prime}} \Theta-\frac{1}{4} \varepsilon_{a b} \varepsilon_{a^{\prime} b^{\prime}} \nabla_{f f^{\prime}} \Theta \nabla^{f f^{\prime}} \Theta\right) \text {, } \\
& \Omega \rightarrow \Theta \Omega, \\
& \Sigma_{a a^{\prime}} \rightarrow \Sigma_{a a^{\prime}}+\Omega \Theta^{-1} \nabla_{a a^{\prime}} \Theta, \\
& s \rightarrow \Theta^{-1} s+\frac{1}{4} \Theta^{-2} \Omega \nabla_{f f^{\prime}} \nabla^{f f^{\prime}} \Theta+\frac{1}{2} \Theta^{-3} \Omega \nabla_{f f^{\prime}} \Theta \nabla^{f f^{\prime}} \Theta \\
& +\Theta^{-2} \Sigma^{f f^{\prime}} \nabla_{f f^{\prime}} \Theta
\end{aligned}
$$

The solution $t$ will be transformed into a solution of (3.1)-(3.8) under these transformations if $\Lambda$ is transformed appropriately. 
Let $\hat{F}^{\mu}=\widehat{F}^{\mu}, \hat{F}_{a b}=\hat{F}_{(a b)}, \hat{\Lambda}=\hat{\Lambda}$ be arbitrary functions defined on $\mathbb{R}^{4}$. It will now be shown that there exist transformations of the types listed above, under which $t$ is transformed into a solution $\hat{t}$ of (3.1)-(3.8) with Ricci-scalar given by $24 \hat{\Lambda}$, such that the coordinate and frame gauge sources obtained for $\hat{t}$ are just $\hat{F}^{\mu}, \widehat{F}_{a b}$ and that in particular Eqs. (3.11)-(3.15) (with ${ }^{\wedge}$ on all quantities) will be satisfied by $\hat{t}$ and the source fields $\hat{\Lambda}, \hat{F}^{\mu}, \hat{F}_{a b}$.

The conformal factor $\Theta$ and the coordinate transformation $\hat{x}^{\mu}\left(x^{\nu}\right)$ are obtained near $S$ by solving on the space time provided by $t$ the following standard Cauchy problem:

$$
\begin{gathered}
\left.\Theta\right|_{S}=1,\left.\quad \frac{\partial \Theta}{\partial x^{\mu}}\right|_{S}=0 ;\left.\quad \hat{x}^{\mu}\left(x^{v}\right)\right|_{S}=x^{\mu},\left.\quad \frac{\partial \hat{x}^{\mu}}{\partial x^{v}}\right|_{S}=\delta^{\mu}{ }_{v}, \\
\nabla_{\lambda} \nabla^{\lambda} \Theta=4\left(\Theta \Lambda-\Theta^{3} \hat{\Lambda}\left(\hat{x}^{\nu}\right)\right), \\
\nabla_{\lambda} \nabla^{\lambda} \hat{x}^{\mu}=2 \Theta^{2} \hat{F}^{\mu}\left(\hat{x}^{v}\right)-2 \Theta^{-1} \nabla_{\lambda} \Theta \nabla_{\lambda} \hat{x}^{\mu} .
\end{gathered}
$$

The first equation is (3.9), where the scalar $\Lambda\left(\Theta^{2} g\right)$ is given in such a way that in the new coordinate system $\hat{x}^{\mu}$ one will have $\Lambda\left(\Theta^{2} g\right)=\hat{\Lambda}$. The second equation is just $\hat{\nabla}_{\lambda} \hat{\nabla}^{\lambda} \hat{x}^{\mu}=2 \hat{F}^{\mu}\left(\hat{x}^{v}\right)$, where $\hat{\nabla}$ denotes the Levi-Civita connection with respect to $\hat{g}_{\mu v}=\Theta^{2} g_{\mu v}$. The rotation of the frame is now determined near $S$ by solving the initial value problem

$$
\left.t_{b}^{a}\right|_{S}=\varepsilon_{b}^{a},\left.\quad \frac{\partial t_{b}^{a}{ }_{b}}{\partial x^{\mu}}\right|_{S}=0, \quad \hat{\nabla}^{\lambda}\left(\left(\hat{\nabla}_{\lambda} t_{a}^{c}\right) \varepsilon_{c d} t_{b}^{d}\right)=\hat{F}_{a b}\left(\hat{x}^{\mu}\right) .
$$

the existence of the unique solutions of these initial value problems can be inferred from the results given in [6] or [11], after transforming the wave equations into symmetric hyperbolic systems.

The initial data chosen in the construction of the transformations and the equation for the conformal factor imply first, that on $S$

$$
n^{a a^{\prime}} n^{b b^{\prime}} \nabla_{a a^{\prime}} \nabla_{b b^{\prime}} \Theta=\nabla_{a a^{\prime}} \nabla^{a a^{\prime}} \Theta=4(\Lambda-\hat{\Lambda}),
$$

hence

$$
\nabla_{a a^{\prime}} \nabla_{b b^{\prime}} \Theta=4(\Lambda-\hat{\Lambda}) n_{a a^{\prime}} n_{b b^{\prime}},
$$

where $n=n^{a a^{\prime}} e_{a a^{\prime}}$ is a unit normal of $S$, and then that

$$
\hat{t}=\left(\hat{e}^{\mu}{ }_{a a^{\prime}}, \hat{\Gamma}_{a a^{\prime} b c}, \hat{\varphi}_{a b c d}, \hat{\phi}_{a b a^{\prime} b^{\prime}}, \hat{\Omega}, \hat{\Sigma}_{a a^{\prime}}, \hat{S}\right)
$$

is related to $t$ on $S$ by

$$
\begin{aligned}
\hat{e}^{\mu}{ }_{a a^{\prime}} & =e^{\mu}{ }_{a a^{\prime}}, \hat{\Gamma}_{a a^{\prime} b c}=\Gamma_{a a^{\prime} b c}=, \hat{\varphi}_{a b c d}=\varphi_{a b c d}, \\
\hat{\phi}_{a b a^{\prime} b^{\prime}} & =\phi_{a b a^{\prime} b}-\left(4 n_{a a^{\prime}} n_{b b^{\prime}}-\varepsilon_{a b} \varepsilon_{a^{\prime} b^{\prime}}\right)(\Lambda-\hat{\Lambda}), \\
\hat{\Omega} & =\Omega, \hat{\Sigma}_{a a^{\prime}}=\Sigma_{a a^{\prime}}, \hat{s}=s+\Omega(\Lambda-\hat{\Lambda})
\end{aligned}
$$

Initial data for the conformal vacuum field equations which are related to each other in this way will be said to belong to the same "conformal class of initial 
data." Notice that unless $\Lambda=\hat{\Lambda}$ on $S$, the rescaling (iii) necessarily implies a transformation of the initial data for (3.1)-(3.8), respectively (3.11)-(3.15). Of course, in the above construction of $\hat{x}^{\mu}, \Theta, t^{a}{ }_{b}$ more general initial conditions could have been given. This would have led to a more complicated relation between the initial data implied by $t$ and $\hat{t}$ on $S$, but these initial data would still belong to the same conformal class. The particular construction above shows that after a new choice of coordinate and frame gauge source the reduced Eqs. (3.11)-(3.15) can be solved with unchanged initial data and the solution will still satisfy the constraints. Since solutions of (3.11)-(3.15) are uniquely determined by the initial data on $S$, the situation described above and the proposition allow one to conclude:

Suppose $t$ is a solution of (3.11)-(3.15) with gauge sources $F^{\mu}, F_{a b}, \Lambda$ and initial data $t_{0}$ on $S$, such that $t$ satisfies (3.1)-(3.8) on $S$ and suppose $\hat{t}$ is a solution of (3.11)-(3.15) (with ${ }^{\wedge}$ on all quantities) with some gauge sources $\hat{F}^{\mu}, \hat{F}_{a b}, \hat{\Lambda}$ and initial data $\hat{t}_{0}$ on $S$, such that $t_{0}$ and $\hat{t}_{0}$ belong to the same conformal class. Then $t$ and $\hat{t}$ are in fact solutions of the conformal vacuum field equations (3.1)-(3.8) which can be transformed into each other by transformations of the types listed above. In particular the solutions $\Omega^{-2} g_{\mu v}$ and $\hat{\Omega}^{-2} \hat{g}_{\mu v}$ of Einstein's vacuum field equations, obtained from $t$, respectively $\hat{t}$, are isometric.

Hence, provided the data are conformally related, solutions of (3.11)-(3.15) for different gauge sources belong to the same conformal class, while in the case of the vacuum field equations the isometry class of a solution of the reduced Eqs. (3.11)(3.13) (with $\Omega \equiv 1, \phi_{a b a^{\prime} b^{\prime}} \equiv 0, \Lambda \equiv 0$ ) is independent of the choice of source functions $F^{\mu}, F_{a b}$.

Proof of the Proposition. By the assumption made above on $\nabla_{a a^{\prime}}$, one has $\overline{\nabla_{a a^{\prime}} c_{b b^{\prime}}}=\nabla_{a a^{\prime}} \bar{c}_{b b^{\prime}}$ in formal calculations. Subtracting from (3.11) suitably the complex conjugates of these equations, one gets a linear homogeneous symmetric hyperbolic system for $e^{\mu}{ }_{a a^{\prime}}-\bar{e}^{\mu}{ }_{a a^{\prime}}$ with the same principal symbol as that of Eq. (3.11). Since the determinant of the symbol is given by $\frac{1}{2} g^{\mu \nu} \xi_{\mu} \xi_{\nu}\left(\xi_{\lambda}\left(e_{00^{\prime}}^{\lambda}+\bar{e}_{00^{\prime}}^{\lambda}+e_{11^{\prime}}^{\lambda}+\bar{e}_{11^{\prime}}^{\lambda}\right)\right)^{2}$ and $e_{a a^{\prime}}^{\mu}=\bar{e}_{a a^{\prime}}^{\mu}$ on the initial surface, the uniqueness property of the equations implies that $e_{a a^{\prime}}^{\mu}=\bar{e}_{a a^{\prime}}$ in the domain of dependence of the initial surface.

Addition of the first and the last equation in (3.11), respectively in (3.12), yields the result that $F^{\mu}, F_{a b}$ is indeed given by (2.6), respectively (2.11). Defining now with $e^{\mu}{ }_{a a^{\prime}}, \Gamma_{a a^{\prime} b c}$ as obtained from $t$ the tensors $t_{a a^{\prime}}{ }^{b b^{\prime}}{ }_{c c^{\prime}}, r_{a b c c^{\prime} d d^{\prime}}$ by (2.3), (2.7), one finds that (3.11), (3.12) are just the equations

$$
\begin{aligned}
-t_{10}^{h h^{\prime}}-t_{1^{\prime} 0^{\prime}}^{h h^{\prime}} & =0, & -\rho^{a b}{ }_{10}-\rho^{a b}{ }_{1^{\prime} 0^{\prime}} & =0, \\
t_{11}^{h h^{\prime}}-t_{0^{\prime} 0^{\prime}}^{h h^{\prime}} & =0, & \rho^{a b}{ }_{11}-\rho^{a b}{ }_{0^{\prime} 0^{\prime}} & =0, \\
-t_{00}^{h h^{\prime}}+t_{1^{\prime} 1^{\prime}}^{h h^{\prime}} & =0, & -\rho^{a b}{ }_{00}-\rho^{a b}{ }_{1^{\prime} 1^{\prime}} & =0, \\
t_{01}^{h h^{\prime}}+t_{0^{\prime} 1^{\prime}}^{h h^{\prime}} & =0, & \rho^{a b}{ }_{01}-\rho^{a b}{ }_{0^{\prime} 1^{\prime}} & =0,
\end{aligned}
$$

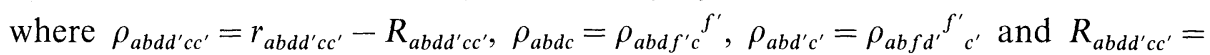
$-\Omega \varphi_{a b c d^{\prime} \varepsilon^{\prime} c^{\prime}}-\phi_{a b d^{\prime} c^{\prime}} \varepsilon_{d c}+\Lambda \varepsilon_{d^{\prime} c^{\prime}}\left(\varepsilon_{a d} \varepsilon_{b c}+\varepsilon_{a c} \varepsilon_{b d}\right)$. One has to show that the following quantities, which are calculated from $t$, will be called "zero-quantities," and denoted collectively by $z$, vanish in the domain of dependence of the initial 
surface:

$$
\begin{aligned}
& t_{c d}^{h h^{\prime}}, t_{c^{\prime} d^{\prime}}^{h h^{\prime}}, \rho_{a b d c}, \rho_{a b d^{\prime} c^{\prime}}, \\
& h_{a b c d^{\prime}}:=\nabla_{d^{\prime}}^{f} \varphi_{a b c f}, L_{a b c d^{\prime}}=\nabla_{{ }^{\prime}}^{f^{\prime}} \phi_{b c d^{\prime} f^{\prime}}-\varphi_{a b c f} \Sigma_{d^{\prime}}^{f}+2 \varepsilon_{a(c} \nabla_{b) d^{\prime}} \Lambda, \\
& R_{a^{\prime} b^{\prime} c^{\prime} d}:=\nabla_{a^{\prime}}^{f} \phi_{f a b^{\prime} c^{\prime}}-\bar{\varphi}_{a^{\prime} b^{\prime} c^{\prime} f^{\prime}} \bar{\Sigma}_{d}^{f^{\prime}}+2 \varepsilon_{a^{\prime}\left(c^{\prime}\right.} \nabla_{\left.b^{\prime}\right) d} \Lambda, \\
& \Delta_{a b a^{\prime} b^{\prime}}:=\phi_{a b a^{\prime} b^{\prime}}-\bar{\phi}_{a b a^{\prime} b^{\prime}, \Delta^{\Omega}} \Omega^{\prime}=\Omega-\bar{\Omega}, \Delta_{b b},:=\Sigma_{b b^{\prime}}-\bar{\Sigma}_{b b^{\prime}}, \\
& k_{b b^{\prime}}:=\nabla_{b b^{\prime}} \Omega-\Sigma_{b b^{\prime}}, \quad q_{b b^{\prime} c c^{\prime}}:=\nabla_{b b^{\prime}} \Sigma_{c c^{\prime}}+\Omega \phi_{b c b^{\prime} c^{\prime}}-s \varepsilon_{b c} \varepsilon_{b^{\prime} c^{\prime}}, \\
& p_{b b^{\prime}}:=\nabla_{b b^{\prime}} s+\phi_{b c b^{\prime} c^{\prime}} \Sigma^{c c^{\prime}}+2 \Lambda \Sigma_{b b^{\prime}}+\Omega \nabla_{b b^{\prime}} \Lambda, \quad \Delta^{s}:=s-\bar{s} .
\end{aligned}
$$

The argument is essentially the same as that used in [1]. For those linear combinations of zero-quantities, which do not vanish already because of (3.11)-(3.15), a linear homogeneous symmetric hyperbolic system of "subsidiary equations" can be derived, which has the uniqueness property in the domain of dependence of the initial surface. This will imply that the zero-quantities vanish there bacause they vanish on the initial surface. Since Eqs. (3.13)-(315) are up to the $\Lambda$-terms essentially those discussed in [1], the derivation of the subsidiary system for the corresponding zero quantities (3.18) is similar to that given in [1] and will not be repeated here. Therefore only the subsidiary system required for the quantities (3.17) will be derived.

From (3.16) and the symmetries of the torsion and curvature tensors one finds that the quantities (3.17) can be expressed in terms of $t_{00}^{h h^{\prime}}, t_{1^{\prime} 0^{\prime}}^{h h^{\prime}}-t_{10}^{h h^{\prime}}, t_{11}^{h h^{\prime}}$, $\rho_{a b 00}, \rho_{a b 11}$, and $\rho_{a b 0^{\prime} 1^{\prime}}-\rho_{a b 01}$, and that it is sufficient to derive a subsidiary system for these quantities.

The Bianchi-identity

$$
\sum_{(j l i)} \nabla_{j} t e^{k} i=\sum_{(j l i)}\left(r^{k}{ }_{i j l}+t_{j i}^{m} t_{m}{ }^{k} l\right)
$$

where the sum over $(j k l)$ denotes sum over cyclic permutation on $j, k, l$ holds for any metric and metric connection. Writing the curvature tensor $r^{k}{ }_{i j l}$ in the form

$$
\varepsilon^{a^{\prime}}{ }_{b^{\prime}}\left(\rho^{a}{ }_{b c c^{\prime} d d^{\prime}}+R_{b c c^{\prime} d d^{\prime}}^{a}\right)+\varepsilon_{b}^{a}\left(\bar{\rho}_{b^{\prime} c c d d^{\prime}}^{a^{\prime}}+\bar{R}_{b^{\prime} c c^{\prime} d d^{\prime}}^{a^{\prime}}\right),
$$

and observing

$$
\sum_{\left(b b^{\prime} c c^{\prime} d^{\prime}\right)}\left(\varepsilon_{a^{\prime} b^{\prime}} R_{a b c c^{\prime} d d^{\prime}}+\varepsilon_{a b} \bar{R}_{a^{\prime} b^{\prime} c c^{\prime} d d^{\prime}}\right)=\varepsilon_{d b \varepsilon^{\prime} \varepsilon_{c^{\prime} d^{\prime}}} \Delta_{a c a^{\prime} b^{\prime}}+\varepsilon_{b^{\prime} d^{\prime}} \varepsilon_{c d} \Delta_{a b a^{\prime} c^{\prime}},
$$

one finds that the right member of (3.19) is in fact homogeneous in the zero quantities. On the other hand one has

$$
\frac{1}{3} \varepsilon^{c^{\prime} e^{\prime}} \varepsilon^{d e}\left(\sum_{\left(e e^{\prime} c c^{\prime} d d^{\prime}\right)} \nabla_{e e^{\prime}} t_{c c^{\prime}}{ }^{h h^{\prime}}{ }_{d d^{\prime}}\right)=\nabla_{d^{\prime}}^{f} t_{c f}^{h h^{\prime}}-\nabla_{c}^{f^{\prime}} t_{f^{\prime} d^{\prime}}^{h h^{\prime}},
$$

which by the preceding remark is equal to an expression ${ }_{d^{\prime} c}^{h^{\prime}}(z)$, homogeneous in $z$. Using (3.16), (3.20), one obtains from (3.19) the equations

$$
\left(\nabla_{00^{\prime}}+\nabla_{11^{\prime}}\right) t_{11}^{h h^{\prime}}+\Gamma_{11^{\prime}}\left(t_{0^{\prime} 0^{\prime}}^{h h^{\prime}}-t_{11}^{h h^{\prime}}\right)-\Gamma_{10^{\prime}}\left(t_{10}^{h h^{\prime}}+t_{1^{\prime} 0^{\prime}}^{h h^{\prime}}\right)=-t_{0^{\prime} 1}^{h h^{\prime}}(z),
$$




$$
\begin{aligned}
& \left(\nabla_{00^{\prime}}+\nabla_{11^{\prime}}\right)\left(t_{1^{\prime} 0^{\prime}}^{h h^{\prime}}-t_{10}^{h h^{\prime}}\right)+\Gamma_{10^{\prime}}\left(t_{00}^{h h^{\prime}}-t_{1^{\prime} 1^{\prime}}^{h h^{\prime}}\right)+\Gamma_{01^{\prime}}\left(t_{11}^{h h^{\prime}}-t_{0^{\prime} 0^{\prime}}^{h h^{\prime}}\right)=\text { th }_{0^{\prime} 0}^{h h^{\prime}}(z)-t_{1^{\prime} 1}^{h h^{\prime}}(z), \\
& \left(\nabla_{00^{\prime}}+\nabla_{11^{\prime}}\right) t_{00}^{h h^{\prime}}+\Gamma_{00^{\prime}}\left(t_{1^{\prime} 1^{\prime}}^{h h^{\prime}}-t_{00}^{h h^{\prime}}\right)-\Gamma_{01^{\prime}}\left(t_{01}^{h h^{\prime}}+t_{0^{\prime} 1^{\prime}}^{h h^{\prime}}\right)=t_{1^{\prime} 0}^{h h^{\prime}}(z),
\end{aligned}
$$

where the notation $\Gamma_{a a^{\prime}}=\nabla_{a a^{\prime}}-e_{a a^{\prime}}$ has been used. This is the desired subsidiary equation for the torsion tensor components. The other subsidiary equations are derived from the second Bianchi-identity

$$
\sum_{(j k l)} \nabla_{j} r^{m}{ }_{i k l}=\sum_{(j k l)} t_{k j}{ }^{h} r^{m}{ }_{i h l} .
$$

The right side of this equation is obviously homogeneous in the zero quantities. After suitable contractions the left side of (3.21) takes the form

which is equal to

$$
\frac{1}{3} \varepsilon^{c^{\prime} e^{\prime}} \varepsilon^{d e}\left(\sum_{\left(e e^{\prime} c c^{\prime} d d^{\prime}\right)}\left(\nabla_{e e^{\prime}} \rho_{a b c c^{\prime} d d^{\prime}}+\nabla_{e e^{\prime}} R_{a b c c^{\prime} d d^{\prime}}\right)\right)
$$

$$
\nabla_{d^{\prime}}^{f} \rho_{a b f c}-\nabla_{c}^{f^{\prime}} \rho_{a b f^{\prime} d^{\prime}}+\Omega h_{a b c d^{\prime}}-L_{c a b d^{\prime}}-k_{d^{\prime}}^{d} \varphi_{a b c d} .
$$

Whence (3.21) implies an equation of the form

$$
\nabla_{d^{\prime}}^{f} \rho_{a b f c}-\nabla_{c}^{f^{\prime}} \rho_{a b f^{\prime} d^{\prime}}=\stackrel{*}{\rho}_{a b c d^{\prime}}(z),
$$

where the right-hand side vanishes if $z=0$. From this and (3.16) ensues the system of subsidiary equations:

$$
\begin{gathered}
\left(\nabla_{00^{\prime}}+\nabla_{11^{\prime}}\right) \rho_{a b 11}+\Gamma_{11^{\prime}}\left(\rho_{a b 0^{\prime} 0^{\prime}}-\rho_{a b 11}\right)-\Gamma_{10^{\prime}}\left(\rho_{a b 10}+\rho_{a b 1^{\prime} 0^{\prime}}\right)=-\stackrel{*}{\rho}_{a b 10^{\prime}}(z), \\
\left(\nabla_{00^{\prime}}+\nabla_{11^{\prime}}\right)\left(\rho_{a b 0^{\prime} 1^{\prime}}-\rho_{a b 01}\right)-\Gamma_{01^{\prime}}\left(\rho_{a b 0^{\prime} 0^{\prime}}-\rho_{a b 11}\right)-\Gamma_{10^{\prime}}\left(\rho_{a b 1^{\prime} 1^{\prime}}-\rho_{a b 00}\right) \\
=\stackrel{*}{\rho}_{a b 00^{\prime}}(z)-\stackrel{*}{\rho}_{a b 11^{\prime}}(z) \\
\left(\nabla_{00^{\prime}}+\nabla_{11^{\prime}}\right) \rho_{a b 00}+\Gamma_{00^{\prime}}\left(\rho_{a b 1^{\prime} 1^{\prime}}-\rho_{a b 00}\right)-\Gamma_{01^{\prime}}\left(\rho_{a b 01}+\rho_{a b 0^{\prime} 1^{\prime}}\right)=\stackrel{*}{\rho}_{a b 01^{\prime}}(z),
\end{gathered}
$$

which together with the equations for $t_{c d}^{h h^{\prime}}$ and for the zero-quantities (3.18) constitutes the subsidiary system.

One may try to use $(2.20)-(2.23)$ to find a similar reduction procedure for Einstein's field equations $R_{\mu \nu}-\frac{1}{2} g_{\mu \nu} R=\kappa T_{\mu \nu}$ with matter fields, without using a third order formalism. The field equations may be written

$$
\begin{aligned}
2 \phi_{a b a^{\prime} b^{\prime}} & =\kappa\left(T_{a b a^{\prime} b^{\prime}}-\frac{1}{4} T \varepsilon_{a b} \varepsilon_{a^{\prime} b^{\prime}}\right), \\
\Lambda & =-24 \kappa T .
\end{aligned}
$$

Using Eqs. (2.21), (2.23) with $t_{c^{\prime} d^{\prime}}^{h h^{\prime}}=0, r_{a b c^{\prime} d^{\prime}}=-\phi_{a b c^{\prime} d^{\prime}}$, one sees that the "geometric part" of the field equations implies a symmetric hyperbolic system for $e_{a a^{\prime}}^{\mu}, \Gamma_{a a^{\prime} b c}$. If from these equations and the way the initial data are given, $e^{\mu}{ }_{a a^{\prime}}=\bar{e}^{\mu}{ }_{a a^{\prime}}$ would follow, then the validity of $\Lambda=-24 \kappa T$ could be inferred from the initial data, the Bianchi identities and the conservation law for the energymomentum tensor. Unfortunately, a way to show that the reality conditions for the frame coefficients will be satisfied does not seem to exist. However, the hyperbolicity of the field equations in a general coordinate system can be shown easily, using the representation of the equations as a second order system for the metric coefficients and the concept of the source functions. 
In an arbitrary coordinate system $x^{\mu}$ the Ricci tensor can be written

$$
R_{\mu \nu}=-\frac{1}{2} g^{\alpha \beta} \frac{\partial^{2} g_{\mu \nu}}{\partial x^{\alpha} \partial x^{\beta}}+\frac{1}{2} g_{\mu \alpha} \nabla_{v} \Gamma^{\alpha}+\frac{1}{2} g_{v \alpha} \nabla_{\mu} \Gamma^{\alpha}+H_{\mu \nu}
$$

where $\Gamma^{\alpha}:=g^{\mu \nu} \Gamma_{\mu \nu}{ }^{\alpha}, \nabla_{\mu} \Gamma^{\alpha}$ is the same expression as that obtained for the covariant derivative of a vector field which with respect to the given coordinate system has components $\Gamma^{\alpha}$, and $H_{\mu \nu}$ is a known function of the metric coefficients and their first order derivatives. For the coordinate gauge source function one finds

$$
2 F^{\mu}\left(x^{\nu}\right)=\nabla_{\lambda} \nabla^{\lambda} x^{\mu}=-\Gamma^{\mu}\left(x^{\nu}\right) .
$$

Considering again this as a given function which just characterizes the gauge, the disturbing second order terms hidden in $\nabla_{v} \Gamma^{\alpha}$, which destroy the "nice" form of the principal part of (3.22), are removed.

Let $F^{\mu}$ be arbitrary real-valued functions defined on $\mathbb{R}^{4}$. By the argument in Sect. 2 we may assume that there exists near some initial surface a coordinate system for which $F^{\mu}$ is the gauge source function. In this coordinate system the geometric part of the field equations will take the form

$$
-\frac{1}{2} g^{\alpha \beta} \frac{\partial^{2} g_{\mu \nu}}{\partial x^{\alpha} \partial x^{\beta}}-g_{\mu \alpha} \nabla_{v} F^{\alpha}-g_{\nu \alpha} \nabla_{\mu} F^{\alpha}+H_{\mu \nu}(g, \partial g)=\kappa\left(T_{\mu \nu}-\frac{1}{2} T g_{\mu \nu}\right) \text {. }
$$

Assume that $g_{\mu \nu}$ is obtained by solving for suitable initial conditions (3.24) together with the equations for the matter fields. Let $R_{\mu \nu}, \Gamma^{\mu}$ be the Ricci tensor and the contracted connection coefficients calculated from $g_{\mu \nu}$. Then (3.22), (3.24) give

$$
R_{\mu \nu}-\kappa\left(T_{\mu \nu}-\frac{1}{2} T g_{\mu \nu}\right)=\frac{1}{2}\left\{g_{\mu \alpha} \nabla_{\nu}\left(\Gamma^{\alpha}+2 F^{\alpha}\right)+g_{\nu \alpha} \nabla_{\mu}\left(\Gamma^{\alpha}+2 F^{\alpha}\right)\right\}
$$

Using this equality to rewrite the equation

$$
0=2 \nabla^{\mu}\left(R_{\mu}{ }^{\nu}-\frac{1}{2} R g_{\mu}{ }^{\nu}-\kappa T_{\mu}{ }^{\nu}\right),
$$

obtained from the Bianchi identity and the conservation law for the energy momentum tensor, one finds

$$
\nabla^{\mu} \nabla_{\mu}\left(\Gamma^{\alpha}+2 F^{\alpha}\right)=R_{\beta}^{\alpha}\left(\Gamma^{\beta}+2 F^{\beta}\right) .
$$

This may be read as a linear homogeneous wave equation for $\Gamma^{\alpha}+2 F^{\alpha}$. Since this type of equation has the uniqueness property one concludes:

Proposition. If $g_{\mu v}$ is a solution of (3.24) together with the matter equations such that on the initial surface one has $\Gamma^{\alpha}+2 F^{\alpha}=0, \nabla_{v}\left(\Gamma^{\alpha}+2 F^{\alpha}\right)=0$, then $g_{\mu v}$ is in fact a solution of Einstein's field equations, since in (3.24) $F^{\alpha}$ may be replaced by $-\frac{1}{2} \Gamma^{\alpha}$.

Remark. Again the detailed discussion of the initial situation, which depends on the type of problem, has been avoided. In a given initial value problem, one will try to arrange the coordinate expression of the initial data to obtain $\Gamma^{\alpha}+2 F^{\alpha}=0$ and to infer the condition $\nabla_{v}\left(\Gamma^{\alpha}+2 F^{\alpha}\right)=0$ from the fact that the data satisfy the constraint equations and $g_{\mu \nu}$ solves (3.24).

Assume that for Eq. (3.24) together with the equations for the matter fields a uniqueness theorem holds. Solving Eq. (2.16) with a new source function $\hat{F}^{\mu}$ for 
a new coordinate system $\hat{x}^{\mu}$ and expressing Eq. (3.24) with respect to these coordinate, one concludes from the uniqueness property that the isometry class of the solution of (3.24) does not depend on the gauge source function.

In forming the reduced propagation equations for the gauge field Eqs. (2.27) one may follow the pattern laid out in the case of the conformal vacuum field equations. One finds that the following system must be satisfied

$$
\begin{aligned}
& 2 \nabla_{00^{\prime}} A_{11^{\prime}}-\nabla_{01^{\prime}} A_{10^{\prime}}-\nabla_{10^{\prime}} A_{01^{\prime}}-B=-\frac{1}{2}\left(\left[A_{1}{ }^{f^{\prime}}, A_{0 f}\right]+\left[A^{f}{ }_{1^{\prime}}, A_{f 0^{\prime}}\right]\right) \\
& -\varphi_{01}-\bar{\varphi}_{0^{\prime} 1^{\prime}} \text {, } \\
& \left(\nabla_{00^{\prime}}+\nabla_{11^{\prime}}\right) A_{10^{\prime}}-\nabla_{10^{\prime}} A_{11^{\prime}}-\nabla_{10^{\prime}} A_{00^{\prime}}=\frac{1}{2}\left(\left[A_{1}{ }^{f^{\prime}}, A_{1 f^{\prime}}\right]-\left[A_{0^{\prime}}^{f}, A_{f 0^{\prime}}\right]\right) \\
& +\varphi_{11}-\bar{\varphi}_{0^{\prime} 0^{\prime}} \text {, } \\
& \left(\nabla_{00^{\prime}}+\nabla_{11^{\prime}}\right) A_{01^{\prime}}-\nabla_{01^{\prime}} A_{00^{\prime}}-\nabla_{01^{\prime}} A_{11^{\prime}}=\frac{1}{2}\left(\left[A_{1^{\prime}}^{f}, A_{f 1^{\prime}}\right]-\left[A_{0}{ }^{f^{\prime}}, A_{0 f^{\prime}}\right]\right) \\
& +\bar{\varphi}_{1^{\prime} 1^{\prime}}-\varphi_{00} \text {, } \\
& 2 \nabla_{11^{\prime}} A_{00^{\prime}}-\nabla_{00^{\prime}} A_{01^{\prime}}-\nabla_{01^{\prime}} A_{10^{\prime}}-B=\frac{1}{2}\left(\left[A_{1}{ }^{f^{\prime}}, A_{0 f^{\prime}}\right]+\left[A^{f}{ }_{1}^{\prime}, A_{f 0^{\prime}}\right]\right) \\
& +\varphi_{01}+\bar{\varphi}_{0^{\prime} 1^{\prime}} \\
& \nabla_{00^{\prime}} \varphi_{11}-\nabla_{10^{\prime}} \varphi_{01}=-\left[A_{0^{\prime}}^{f}, \varphi_{1 f}\right] \text {, } \\
& \left(\nabla_{00^{\prime}}+\nabla_{11^{\prime}}\right) \varphi_{01}-\nabla_{10^{\prime}} \varphi_{11}-\nabla_{01^{\prime}} \varphi_{00}=\left[A_{1^{\prime}}^{f}, \varphi_{1 f}\right]-\left[A_{0^{\prime}}^{f}, \varphi_{0 f}\right] \text {, } \\
& \nabla_{11^{\prime}}, \varphi_{00}-\nabla_{01}, \varphi_{01}=\left[A^{f}{ }_{1}^{\prime}, \varphi_{0 f}\right] \text {. }
\end{aligned}
$$

Here $B$ is a given su $(n)$-valued function on the space time and the functions $A_{a a^{\prime}}$ for the different values of the indices $a a^{\prime}$ are thought of as being independent. Then (3.25), (3.26) is a symmetric hyperbolic system for $A_{a a^{\prime}}, \varphi_{a b}=\varphi_{(a b)}$.

Proposition. Let data $A^{0}{ }_{a a^{\prime}}=\bar{A}^{0}{ }_{a a^{\prime}}, \varphi^{0}{ }_{a b}=\varphi^{0}{ }_{(a b)}$, taking values in su (n), be given on a suitable initial surface, and suppose that $A_{a a^{\prime}}, \varphi_{a b}$ is a solution of (3.25), (3.26) for these data, which is given in the domain of dependence of the initial surface. Then $A_{a a^{\prime}}, F_{a a^{\prime} b b^{\prime}}=\varepsilon_{a^{\prime} b^{\prime}} \bar{\varphi}_{a b}+\varepsilon_{a b} \bar{\varphi}_{a^{\prime} b^{\prime}}$ satisfy Eq. (2.25) everywhere if they satisfy (2.25) on the initial surface.

Remark. Again one finds, following the discussion leading to Eq. (2.32) and using the uniqueness property of the system (3.25), (3.26), that the solutions of the reduced equations for the same data but with different source fields are related by gauge transformations.

The proof of the proposition has the same structure as that in the case of the conformal vacuum field equations. Taking the spinor complex conjugates of Eqs. (3.25) and substracting these suitably from Eqs. (3.25), one obtains a linear homogeneous symmetric hyperbolic system for $A_{a a^{\prime}}-\bar{A}_{a a^{\prime}}$ which allows one to conclude that $A_{a a}=\bar{A}_{a a}$ everywhere, since it holds initially. Adding the first and the last of Eqs. (3.25) one finds that $B$ in fact satisfies Eq. (2.30). Calculating now from the solution $A_{a a^{\prime}} \varphi_{a b}$ the quantities $l_{a a^{\prime} b b^{\prime}}, h_{c^{\prime} c}$ defined by (2.26), one finds that (3.25), (3.26) are indeed the equations 


$$
\begin{array}{rlrl}
-l_{10}-l_{1^{\prime} 0^{\prime}} & =0, & -h_{0^{\prime} 1} & =0, \\
l_{11}-l_{0^{\prime} 0^{\prime}} & =0, & -h_{0^{\prime} 0}+h_{1^{\prime} 1} & =0, \\
-l_{00}+l_{1^{\prime} 1^{\prime}} & =0, & h_{1^{\prime} 0} & =0 . \\
l_{01}+l_{0^{\prime} 1^{\prime}} & =0, &
\end{array}
$$

From this one concludes that Eqs. (2.25) will be satisfied, if it can be shown that $h_{1^{\prime} 1}, l_{00}, l_{11}, l_{0^{\prime} 1},-l_{01}$, denoted collectively by $z$, vanish. Only assuming that $A_{a a^{\prime}}=\bar{A}_{a a^{\prime}}, \varphi_{a b}=\varphi_{(a b)}$, and both fields take values in su $(n)$, one finds by direct calculation that $l_{a a^{\prime} b b^{\prime}}, h_{c^{\prime} c}$ must satisfy the identities

$$
\begin{aligned}
& \frac{1}{3} \varepsilon^{a b} \varepsilon^{a^{\prime} c^{\prime}}\left(\sum_{\left(a a^{\prime} b b^{\prime} c c\right)}\left(\nabla_{a a^{\prime}}, l_{b b^{\prime} c c^{\prime}}-\left[A_{a a^{\prime}}, l_{b b^{\prime} c c^{\prime}}\right]\right)\right)=\bar{h}_{b^{\prime} c}-h_{b^{\prime} c} \\
& 2\left(\nabla^{a a^{\prime}} h_{a^{\prime} a}-\left[A^{a a^{\prime}}, h_{a^{\prime} a}\right]\right)=\left[\varphi^{c f}, l_{c f}\right] .
\end{aligned}
$$

Expressing the left-hand side of the first identity in terms of $l_{a b}, l_{a^{\prime} b^{\prime}}$, one finds

$$
\nabla_{b^{\prime}}^{h} l_{h c}-\nabla_{c}{ }^{h^{\prime}} l_{h^{\prime} b^{\prime}}=\left[A^{h}{ }_{b^{\prime}} l_{h c}\right]-\left[A_{c}^{h^{\prime}}, l_{h^{\prime} b}\right]+\frac{1}{2}\left(\bar{h}_{b^{\prime} c}-h_{b^{\prime} c}\right) \text {. }
$$

One deduces from (3.28), (3.29) equations of the form

$$
\begin{aligned}
\left(\nabla_{00^{\prime}}+\nabla_{11^{\prime}}\right) h_{1^{\prime} 1} & =\stackrel{*}{h}(z), \\
\left(\nabla_{00^{\prime}}+\nabla_{11^{\prime}}\right) l_{11} & =-\stackrel{*}{l}_{0^{\prime} 1}(z), \\
\left(\nabla_{00^{\prime}}+\nabla_{11^{\prime}}\right)\left(l_{0^{\prime} 1^{\prime}}-l_{0^{\prime} 0}\right) & =\stackrel{*}{l}_{0^{\prime} 0}(z)-\stackrel{*}{l}_{1^{\prime} 1}(z), \\
\left(\nabla_{00^{\prime}}+\nabla_{11^{\prime}}\right) l_{00} & =\stackrel{*}{l}_{1^{\prime} 0}(z),
\end{aligned}
$$

with smooth functions $\stackrel{*}{h},{\stackrel{l}{c^{\prime} d}}$ which satisfy $\stackrel{*}{h}(0)=0,{ }_{c^{\prime} d}^{*}(0)=0$. Since, however, $z=0$ initially, these equations imply that $z=0$ everywhere.

Acknowledgements. I should like to thank B. Schmidt and H.-J. Seifert for discussions.

\section{References}

1. Friedrich, H.: The asymptotic characteristic initial value problem for Einstein's vacuum field equations as an initial value problem for a first-order quasilinear symmetric hyperbolic system. Proc. R. Soc. (London) A 378, 401-421 (1981)

2. Friedrich, H.: On the regular and the asymptotic characteristic initial value problem for Einstein's vacuum field equations. Proc. R. Soc. (London) A 375, 169-184 (1981)

3. Friedrich, H.: Cauchy problems for the conformal vacuum field equations in general relativity. Commun. Math. Phys. 91, 445-472 (1983)

4. Friedrichs, K. O.: Symmetric hyperbolic linear differential equations. Commun. Pure Appl. Math. 8, 345-392 (1954)

5. Courant, R., Hilbert, D.: Methods of mathematical physics, Vol. II. New York: Interscience 1962

6. Kato, T.: The Cauchy problem for quasi-linear symmetric hyperbolic systems. Arch. Ration. Mech. Anal. 58, 181-205 (1975)

7. Friedrich, H.: On purely radiative space-times, preprint, Hamburg 1985.

8. Friedrich, H.: On some (con-) formal properties of Einstein's field equations and their consequences. 
In: Proceedings of the conference on "Asymptotic behaviour of mass and space-time geometry." Corvallis 1983, Flaherty, F. J., ed. Berlin Heidelberg, New York: Springer 1984

9. Lanczos, C.: Ein vereinfachendes Koordinatensystem für die Einsteinschen Gravitationsgleichungen. Phs. Z. 23, 537-539 (1922)

10. Choquet-Bruhat, $Y .:$ Théorème d'existence pour certain systèmes d'èquations aux derivées partielles non linéaires. Acta Math. 88, 141-225 (1952)

11. Mizohata, S.: The theory of partial differential equations. Cambridge: Cambridge University Press 1973

Communicated by S. W. Hawking

Received December 11, 1984 
